\title{
Gender Differences in Patients with Anxiety after Coronary Artery Bypass Surgery
}

\author{
Yalcin Guzelhan, MD, ${ }^{1}$ Cenk Conkbayir, $\mathrm{MD},{ }^{2}$ Murat Ugurlucan, $\mathrm{MD},{ }^{3}$ Cenk Eray Yildiz, $\mathrm{MD},{ }^{3}$ \\ Ufuk Alpagut, $\mathrm{MD}^{3}$, Nilgun Bozbuga, $\mathrm{MD}, \mathrm{PhD}^{3}$ \\ ${ }^{1}$ Psychiatry Clinic, Istanbul Education and Research Hospital, Istanbul, Turkey; ${ }^{2}$ Department of Cardiology, Near East \\ University, Nicosia, Cyprus; ${ }^{3}$ Department of Cardiovascular Surgery, Istanbul University Medical Faculty, Istanbul, Turkey
}

\section{ABSTRACT}

Objective: The present study was designed to evaluate the relationship between gender and coexisting anxiety in patients undergoing coronary artery surgery.

Materials and Methods: A total of 137 patients (41 women and 96 men with a mean age $66.1 \pm 6.0$ years) patients underwent state and trait anxiety evaluation at baseline (preoperatively) and at six months after (postoperatively) coronary artery bypass graft (CABG). Anxiety symptoms were assessed at enrollment using the Spielberger State-Trait Anxiety Inventory (STAI). Psychological, social, clinical, and surgical data were assessed statistically.

Results: There were statistically significant differences between female and male patient characteristics for the mean age, mean education year, and mean body mass index. The women were found to be statistically younger and less educated, and more likely to be overweight, diabetic, and hyperlipidemic. The mean hospitalization time, wound infection, and extreme postoperative pain complaints were found to be higher in the female group. 61 patients ( 33 female and 28 male) $(44.5 \%)$ were classified as presenting clinically significant anxiety symptoms (STAI score of $\geq 40$ ). The female patients' STAI scores were significantly higher than men in state and trait anxiety, both preoperatively and six months postoperatively. Postoperatively, there was not any significant decrease in the level of trait anxiety when comparing the level of state anxiety in female patients.

Conclusion: Even after adjusting for known risk factors for compromised STAI, women do not show the same long-term quality benefits of CABG surgery that men do. The results indicate that the STAI is a valuable instrument for identifying and supporting patients with higher levels of anxiety, which can aid in determining patients that may have poor adjustment after CABG surgery.

Received November 13, 2015; received in revised form November 18, 2015; accepted March 21, 2016.

Correspondence: Murat Ugurlucan, Assoc. Prof., MD, Department of Cardiovascular Surgery, Istanbul University Medical Faculty, Millet Caddesi, Capa, 34390 Fatib / Istanbul, Turkey; +90-212-414-20-00, fax: +90-212-

534-22-32 (e-mail: muratugurlucan@yahoo.com).

\section{INTRODUCTION}

Symptoms of anxiety are associated with increased risk of coronary artery disease, and a potentially poor prognosis among patients with existing coronary artery disease in healthy populations [Kubzansky 2006; Suls 2005]. Several studies have shown the importance of psychosocial factors in the development of coronary heart disease (CHD), and/or the worsening of symptoms and survival rates in the presence and persistence of CHD [Hemingway 1999]. In particular, anxiety, depression, and self-rated health seem to influence the postoperative recovery after coronary artery bypass graft (CABG) surgery [Rothenhausler 2005].

A vast amount of literature on depression and cardiovascular disease suggests that depression has a direct effect on coronary pathology [Musselmann 1998]. Some studies have analyzed the influence of phobic anxiety on heart failure, however, the role of anxiety as a personality dimension has rarely been studied [Kawachi 1995]. Few studies have assessed symptoms of both anxiety and depression simultaneously [Friedmann 2006]. It has been shown that the presence of depressive symptoms is associated with progression of atherosclerosis among individuals with previous CABG surgery [Wellenius 2008]. Although anxiety and depression were highly positively correlated in these patients, only anxiety was found to be associated with increased mortality and morbidity.

Among CHD patients undergoing CABG surgery, anxiety was shown to be common, and was associated with an increased all-cause mortality risk [Székely 2007; Rymaszewska 2003]. Trait anxiety was found to be significantly higher in patients that were hospitalized with arrhythmia, congestive heart failure, or myocardial infarction during a four-year period after CABG and heart valve surgery [Székely 2007].

Improved quality of life is one of the most important outcomes of cardiac surgery [Hunt 2000; Duits 1997; Burker 1995]. However, not all patients experience improvements after surgery. Despite good surgical results, some patients report poor health status and need frequent hospitalization after coronary artery surgery. Postoperative quality of life after cardiac operations has become an important measure of the effectiveness of the CABG surgery. Important predictors of improved quality of life outcomes have been shown to include age, education, comorbidities, and gender [Brummett 2001].

Female patients presenting for CABG surgery have different characteristics than male patients. These female patients tend to be older, less educated, and more likely to 
Table 1. Demographics and Operative Variables for Female and Male Patients

\begin{tabular}{lccc}
\hline & $\begin{array}{c}\text { Female } \\
\mathrm{n}=41\end{array}$ & $\begin{array}{c}\text { Male } \\
\mathrm{n}=96\end{array}$ & $P$ \\
\hline Age (year) & $62.6 \pm 6$ & $70.6 \pm 6$ & .02 \\
Education (year) & $7.5 \pm 2.9$ & $10.6 \pm 3.7$ & .04 \\
Ejection fraction (\%) & $52.3 \pm 5.7$ & $48.9 \pm 3.1$ & $\mathrm{~ns}$ \\
BMI & $28.8 \pm 3.3$ & $24.5 \pm 3.8$ & .02 \\
Hypertension & $41.2 \%$ & $38.5 \%$ & \\
DM & $50.4 \%$ & $44.7 \%$ & \\
Hyperlipidemia & $62.3 \%$ & $49.1 \%$ & \\
CPB time (min) & $72.1 \pm 14.8$ & $74.1 \pm 20.2$ & .82 \\
ACC time (min) & $42.5 \pm 7.7$ & $40.8 \pm 7.9$ & .68 \\
No. of GCA & $2.6 \pm 0.3$ & $2.8 \pm 0.5$ & $\mathrm{~ns}$ \\
ICU time (days) & $2.8 \pm 0.6$ & $2.6 \pm 0.4$ & $\mathrm{~ns}$ \\
Hospitalization time (days) & $10.12 .6 \pm 2.7$ & $7.8 \pm 01.8$ & .04 \\
\hline
\end{tabular}

BMI indicates body mass index; DM, diabetes mellitus; CPB, cardiopulmonary bypass; ACC, aortic cross clamping; GCA, grafted coronary artery; $\mathrm{ICU}$, intensive care unit; ns, not significant.

be hypertensive, diabetic, and obese [Bagheri 2014; Moore 1995; Cannistra 1992]. In addition, women are more likely to be anxious and depressed when compared with their male counterparts [Burker 1995].

The present study was designed to determine the effect of anxiety in female and male patients undergoing coronary artery surgery, and to examine the relationship between gender and coexisting anxiety in patients before and after CABG.

\section{MATERIALS AND METHODS}

A total of 137 patients (41 women and 96 men with a mean age $66.1 \pm 6.0$ years) were scheduled for elective coronary artery bypass grafting, and were prospectively enrolled in the study and gave written informed consent.

Specific exclusion criteria included unstable angina, decompensate heart failure, large left ventricular aneurysm, life threatening arrhythmias, life threatening cerebrovascular disease, systemic hypertension refractory to drug therapy, and severe renal or hepatic dysfunction. In addition, patients with major psychiatric disorders or absolute contraindications to treatment with any study medications were excluded.

All patients completed psychometric test batteries. Selfreported anxiety symptom questionnaires were completed preoperatively and six months after discharge. Anxiety symptoms were assessed at enrollment using the Spielberger StateTrait Anxiety Inventory (STAI) in all patients. The STAI consists of two 20-item scales that measure "state" (current) and "trait" (chronic) anxiety [Speilberger 1970]. Data were also collected on social and clinical measures.
Social (education level) and clinical (age, ejection fraction, body mass index, hypertension, diabetes mellitus, hyperlipidemia) risk factors, and perioperative characteristics (cardiopulmonary bypass time, aortic cross-clamping time, number of bypassed grafts, intensive care unit time, hospitalization time) were recorded for each patient and compared statistically between the two gender groups (Table 1).

Postoperative variables (postoperative myocardial infarction, low cardiac output, congestive heart failure, cerebrovascular accident, arrhythmia renal and pulmonary complications) were recorded.

\section{Measurement of Anxiety}

The two STAI sub-scores, state (STAI-S) and trait (STAI$\mathrm{T})$, have high concordance in both people with diagnosed generalized anxiety disorder and those without diagnosed anxiety. The baseline measurement of the state and trait anxiety levels (STAI-S and STAI-T) were taken before surgery; patients also took STAIs upon completion of the session after six months postoperation.

The STAIs do not capture information on patients' clinical or treatment history. Representative items include statements such as "I feel nervous" and "I feel worried." These items are rated on a 4-point scale of how well they describe the patient's current or typical mood from "not at all" to "very much so." STAI scores range from 20 to 80, with higher scores indicating more severe symptoms and greater anxiety. We accepted the STAI scores of $<40$ to indicate no or minimal symptoms, and $\geq 40$ to indicate the presence of moderate or severe symptoms.

\section{Surgical Management}

In elective CABG operations, anesthetic management with midazolam, fentanyl, isoflurane, and vecuronium was performed. All patients underwent CABG using open heart surgery technique via standard median sternotomy in one center. After harvesting the left internal mammary artery (LIMA) and autologous saphenous vein graft (SVG), cardiopulmonary bypass (CPB) was performed by cannulation of the ascending aorta and vena cava through right atrial auricula. All patients underwent standard nonpulsatile moderate hypothermic $\left(32^{\circ} \mathrm{C}\right) \mathrm{CPB}$. Nonpulsatile perfusion of 2 to 2.4 liters per minute per square meter was maintained throughout surgery. All patients underwent aortic cross-clamping to achieve cardioplegic arrest. Myocardial protection was provided by continuous retrograde isothermic blood cardioplegia through the coronary sinus. Throughout $\mathrm{CPB}$, arterial partial pressure of carbon dioxide was maintained at 35 to $40 \mathrm{~mm}$ $\mathrm{Hg}$, and partial pressure of oxygen at 150 to $200 \mathrm{~mm} \mathrm{Hg}$.

All patients received LIMA and SVGs, performing routine anasthomosis technique. The majority of patients $(82.2 \%)$ received more than two coronary vessels grafting to the left and right coronary system (left anterior descending artery, right coronary artery and/or circumflex artery). The mean bypassed coronary arteries of the patients was $2.7 \pm 0.4$ vessels. No patients in either gender group underwent urgent or complicated associated cardiac operation. No patients required mechanical left ventricular support postoperatively. 
Table 2. Gender Differences for STAI Score at Baseline and 6-month Follow-up

\begin{tabular}{lccc}
\hline STAI & $\begin{array}{c}\text { Female } \\
\mathrm{n}=41\end{array}$ & $\begin{array}{c}\text { Male } \\
\mathrm{n}=96\end{array}$ & $P$ \\
\hline & & & \\
Baseline & & & \\
STAI-S & $59.4 \pm 7.7$ & $46.4 \pm 7.3$ & .0001 \\
STAI-T & $55.5 \pm 8.6$ & $45.1 \pm 4.0$ & .002 \\
6-month Follow-up & & & \\
STAI-S & $52.2 \pm 9.6$ & $42.8 \pm 6.1$ & .002 \\
STAI-T & $52.5 \pm 6.9$ & $42.5 \pm 4.6$ & .001 \\
\hline
\end{tabular}

STAI-S indicates State-Trait Anxiety Inventory "state" (current); STAI-T, State-Trait Anxiety Inventory "trait" (chronic).

\section{Statistical Analysis}

Psychological, social, and surgical data were assessed. For descriptive purposes, baseline univariate comparisons were performed to identify gender-related differences for each STAI. Similar comparisons were performed for the six-month follow-up time periods.

Statistical analysis was performed using the computer software SPSS 20.0 for windows (SPSS Inc., Chicago, IL). Data are expressed as mean values \pm standard deviation for continuous variables and as numbers with percentage for categorical variables. The discrete variables were compared using the $\chi^{2}$ test, and continuous variables were compared using a two-tailed t test. Univariate analysis and logistic regression analysis were performed to identify the potential risk factors associated with anxiety. A $P$ value of .05 or less was considered to be significant.

\section{RESULTS}

The mean age of 137 patients was $66.1 \pm 6$ years. The patients were divided and compared between gender groups (41 females and 96 males). There was a statistically significant difference between female and male patient characteristics in the mean age, mean education year, and mean body mass index. The women were found to be younger, less educated, and more likely to be overweight, diabetic, and hyperlipidemic, statistically. Although the mean intensive care unit period was similar, the extended hospitalization period was found statistically higher in female group $(P=.04)$ (Table 1).

There were no mortalities. The rate of morbidity (postoperative myocardial infarction, low cardiac output, congestive heart failure, cerebrovascular accident, arrhythmia, renal and pulmonary complication) after bypass surgery were similar between the women and the men, with the exception of the rate of wound infection $(P=.05)$. The high wound infection rate in female patients could be related to the higher diabetic disposition of the female group.
61 patients (44.5\%), 33 females and 28 males, were classified as presenting clinically significant anxiety symptoms (STAI score $\geq 40$ ). The preoperative and postoperative repeated STAI measure analysis showed significant gender effects on state and trait anxiety. A significant relationship between the preoperative and postoperative state and trait anxiety levels was found in both gender groups.

The incidence of higher postoperative anxiety levels was significantly higher in the patients who had preoperative STAI scores of $\geq 40$ points. Preoperative STAI-S was significantly higher than any of the postoperative STAI values in both gender groups. On the contrary, postoperative STAI-T scores remained relatively consistent when compared with the preoperative value.

Table 2 presents the means for all measures, for males and females, for each test period. The female patients' STAI scores in state and trait anxiety were significantly higher than those of men, both preoperatively and six months postoperatively. There was no significant decrease in the level of trait anxiety when comparing the level of state anxiety in the female patients during the six-month follow-up.

\section{DISCUSSION}

The long-term negative influence of negative psychosocial stressors increases the severity of cardiovascular diseases [Kubzansky 2006; Rothenhausler 2005; Suls 2005; Hemingway 1999; Musselmann 1998; Kawachi 1995]. However, the severity or progression of the cardiovascular diseases is difficult to measure by traditional clinical variables, such as hypertension, peripheral vascular diseases, or diabetes, because these categories do not provide any information about the duration and/or stage of these diseases.

Chronic stress provokes myocardial infarction in people with underlying heart disease through a number of proposed mechanisms, including vasomotor abnormalities in epicardial coronary arteries, abnormally high production of catecholamines in response to stress resulting in higher myocardial oxygen demand and risk of sudden death, and abnormalities in thrombosis and homeostasis [Friedmann 2006; Hemingway 1999]. Anxiety could indirectly alter control of other clinical risk factors through its effects on weight, smoking, alcohol use, and exercise. Alternatively, anxiety may be a risk marker of other psychiatric states or more severe underlying disease.

There is convincing evidence to support the hypothesis that an association between psychosocial risks and pathophysiological alterations exists. Dysregulation of the pituary-adrenal axis, sympathoadrenal hyperactivity, alterations in autonomic nervous system activity, alteration in platelet receptors and reactivity, and immunological changes have been reported [Hemingway 1999; Musselmann 1998; Kawachi 1995]. Autonomic dysregulation may explain the high incidence of arrhythmia in anxious patients. This pathophysiological alteration, and the decreased heart rate variability as a consequence, increase the risk for ventricular tachycardia, fatal arrhythmias, and sudden death [Tully 2008; Friedmann 2006; Kawachi 1995]. 
The effect of anxiety in patients undergoing coronary artery surgery, particularly women, may be under recognized, and warrants increased attention [Cebeci 2011]. Therefore, we examined the effect of anxiety as a risk factor for the outcomes of CABG surgery in men and women by adjusting for baseline differences. Our results indicate that postoperative anxiety levels are attributable to preoperative anxiety levels, not to differential recovery after CABG surgery. The female patients showed significantly worse outcomes in state and trait anxiety than the male patients at a six-month followup. After adjusting for baseline differences, the women are at greater risk for increased anxiety.

There has been some disagreement in the literature as to whether women fare worse than men do after CABG surgery. Rankin found that women were more likely to die during hospitalization, stay longer in intensive care units, and experience cardiac dysfunction [Rankin 1990]. Some studies have reported that men experience improved outcomes when compared with women [Steine 1996; Higgins 1992]. However, Allen and colleagues have reported that long-term outcomes for men and women are similar [Allen 1997].

One factor that may explain this apparent discrepancy is that postoperative recovery differences may reflect the preoperative differences; women appear to do worse because they were initially more compromised than men [King 2000; Cronin 1997; Hawthorne 1994]. In fact, Jacobs reports that although raw incidence rates show worse outcomes for women than men, after accounting for preexisting differences, female gender is actually a predictor of improved five-year survival after percutaneous transluminal coronary angioplasty or CABG [Jacobs 1998].

A relationship between anxiety and pain in patients undergoing CABG has been reported [Greszta 2008]. It has been also observed that patients with a high level of preoperative state anxiety and stronger trait anxiety respond worse to analgesic medication than patients with a low level of state and trait anxiety. Therefore, actions that are undertaken to reduce patients' anxiety may reduce patients' need of analgesic medications [Cebeci 2011].

\section{CONCLUSION}

This study demonstrated gender differences in STAI scores, with females scoring worse. The differences persist even after controlling for baseline measures. These findings indicate that these postoperative gender differences in STAI are not explained by pre-existing differences. Gender differences exist at each time point, with women showing more anxiety. Even after adjusting for known risk factors for compromised STAI, women do not show the same longterm quality benefits of CABG surgery that men do. The results indicate that the STAI is a valuable instrument for identifying patients that are at risk for poor adjustment after CABG surgery.

The results of the present study suggest that the assessment of anxiety could help in risk stratification, and identification of patients at risk of mortality and cardiovascular morbidity after cardiac surgery. Postoperative six-month STAI scores were found more predictive for cardiovascular events compared with the preoperative values. We considered trait anxiety as a more reliable indicator of individual differences in anxiety symptoms. The early postoperative period of selected nursing interventions on mood and anxiety of patients undergoing CABG may be influenced the quality of life improvement after coronary surgery.

Furthermore, the assessment of preoperative well-being should be integrated into routine care in order to identify and support patients with higher levels of anxiety. Appropriate therapeutic interventions may consequently be developed and evaluated in future research. However, future studies on coronary heart disease populations that measure anxiety variability will be needed to further explicate the possible mechanisms responsible for the association between anxiety and mortality and morbidity.

\section{REFERENCES}

Allen JK, Xu X. 1997. Coronary revascularization in women. Crit Care Nurs Clin North Am 9:497-509.

Bagheri J, Sarzaeem MR, Valeshabad AK, et al. 2014. Effect of sex on early outcomes of isolated coronary artery bypass grafting. Turk Gogus Kalp Dama 22:534-9.

Brummett BH, Barefoot JC, Siegler IC, et al. 2001. Characteristics of socially isolated patients with coronary artery disease who are at elevated risk for mortality. Psychosom Med 63:267-72.

Burker EJ, Blumenthal JA, Feldman M, et al. 1995. Depression in male and female patients undergoing cardiac surgery. British J Clin Psych 34:119-28.

Cannistra LB, Balady GJ, O’Malley CJ, et al. 1992. Comparison of the clinical profile and outcome of women and men in cardiac rehabilitation. Am J Cardiol 69:1274-9.

Cebeci F, Celik SS. 2011. Effect of discharge teaching and counselling on anxiety and depression level of coronary artery bypass graft patients. Turk Gogus Kalp Dama 19:176-9.

Cronin SN, Logsdon C, Miracle V. 1997. Psychosocial and functional outcomes in women after coronary artery bypass surgery. Crit Care Nurse 17:19-24.

Duits AA, Boeke S, Taams MA, et al. 1997. Prediction of quality of life after coronary artery bypass graft surgery: a review and evaluation of multiple, recent studies. Psychosom Med 59:257-68.

Friedmann E, Thomas SA, Liu F, et al. 2006. Sudden cardiac death in heart failure trial investigators. Relationship of depression, anxiety, and social isolation to chronic heart failure outpatient mortality. Am Heart J 152:940.e1-8.

Greszta E, Sieminska MJ. 2008. Relationship of preoperative anxietystate and anxiety-trait in patients qualified for coronary artery bypass graft surgery to the perception of postoperative pain and other pain complaints. Ann Acad Med Stetin 54:157-63.

Hawthorne MH. 1994. Gender differences in recovery after coronary artery surgery. Image J Nurs Sch 26:75-80.

Hemingway H, Marmot M. 1999. Evidence based cardiology: psychosocial factors in the aetiology and prognosis of coronary heart disease: systematic review of prospective cohort studies. BMJ 318:1460-7.

Higgins TL, Estafanous FG, Loop FD, et al. 1992. Stratification of 
morbidity and mortality outcome by preoperative risk factors in coronary artery bypass patients. A clinical severity score. JAMA 267:2344-8.

Hunt JO, Hendrata MV, Myles PS. 2000. Quality of life 12 months after coronary artery bypass graft surgery. Heart Lung 29:401-11.

Jacobs AK, Kelsey SF, Brooks MM, et al. 1998. Better outcome for women compared with men undergoing coronary revascularization: a report from the bypass angioplasty revascularization investigation (BARI). Circulation 98:1279-85.

Kawachi I, Sparrow D, Vokonas PS, et al. 1995. Decreased heart rate variability in men with phobic anxiety (data from the normative aging study). Am J Cardiol 75:882-5.

King KM. 2000. Gender and short-term recovery from cardiac surgery. Nurs Res 49:29-36.

Kubzansky LD, Cole SR, Kawachi I, et al. 2006. Shared and unique contributions of anger, anxiety, and depression to coronary heart disease: A prospective study in the normative aging study. Ann Behav Med 31:21-9.

Moore SM. 1995. A comparison of women's and men's symptoms during home recovery after coronary artery bypass surgery. Heart Lung 24:495-501.

Musselmann DL, Evans D, Nemeroff CB. 1998. The relationship of depression to cardiovascular disease. Arch Gen Psychiatr 55:580-92.

Rankin SH. 1990. Differences in recovery from cardiac surgery: a profile of male and female patients. Heart Lung 19:481-5.

Rothenhausler HB, Grieser B, Nollert G, et al. 2005. Psychiatric and psychosocial outcome of cardiac surgery with cardiopulmonary bypass: a prospective 12-month follow-up study. Gen Hosp Psychiatry 27:18-28.

Rymaszewska J, Kiejna A, Hadrys T. 2003. Depression and anxiety in coronary artery bypass grafting patients. Eur Psychiatry 18:155-60.

Speilberger CG, Lushene R. 1970. State-Trait Anxiety Inventory manual. Palo Alto, CA: Consulting Psychologists Press.

Steine S, Laerum E, Eritsland J, et al. 1996. Predictors of enhanced wellbeing after coronary artery bypass surgery. J Intern Med 239:69-73.

Suls J, Bunde J. 2005. Anger, anxiety, and depression as risk factors for cardiovascular disease: The problems and implications of overlapping affective dispositions. Psychol Bull 131:260-300.

Székely A, Balog P, Benkö E, et al. 2007. Anxiety predicts mortality and morbidity after coronary artery and valve surgery a 4-year follow-up study. Psychosom Med 69:625-31.

Tully PJ, Baker RA, Knight JL. 2008. Anxiety and depression as risk factors for mortality after coronary artery bypass surgery. J Psychosom Res 64:285-90.

Wellenius GA, Mukamal KJ, Kulshreshtha A, et al. 2008. Depressive symptoms and the risk of atherosclerotic progression among patients with coronary artery bypass grafts. Circulation 117:2313-9. 